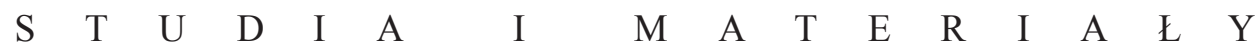

Marzena Liedke

ORCID: https://orcid.org/0000-0002-4054-098X

Uniwersytet w Białymstoku, Wydział Historii i Stosunków Międzynarodowych email: mliedke@uwb.edu.pl

\title{
Death of the Magnate: Life Expectancy of the Magnatery in the Polish-Lithuanian Commonwealth Versus the Demography of the Western European Elites in the Early Modern Age*
}

\section{Śmierć magnata. Oczekiwana długość życia magnaterii w Rzeczypospolitej Obojga Narodów a demografia elit zachodnioeuropejskich w epoce wczesnonowożytnej}

\begin{abstract}
The aim of the article is to present life expectancy calculations for magnates, the elite of the nobility (but not an exact equivalent of European aristocracy) from the Polish-Lithuanian Commonwealth in the 16th-18th centuries (life tables for women and men). The paper also contains a comparison with the results previously obtained for Western European aristocracy. The author tried to verify if there were
\end{abstract}

\begin{abstract}
Abstrakt
Celem artykułu jest zaprezentowanie obliczeń oczekiwanej długości życia magnaterii - elity stanu szlacheckiego Rzeczypospolitej Obojga Narodów w XVI-XVIII wieku (skonstruowano tablice trwania życia dla kobiet i mężczyzn na podstawie bazy danych uwzględniającej informacje genealogiczne odnośnie do 8 rodów koronnych i 9 litewskich). Zamieszczono w nim też porównanie uzyskanych
\end{abstract}

* This paper was produced as part of the project Religious Conditions of Economic and Demographic Development in the Preindustrial Period - the case of the Polish-Lithuanian Commonwealth no. 2016/23/B/HS3/03050 financed by the National Science Centre, Poland. The author would like to thank Piotr Guzowski and Radosław Poniat for all their comments and help with the statistical analysis. 
differences in life expectancy between men and women from two parts of the Commonwealth: The Kingdom of Poland and the Grand Duchy of Lithuania. Because only a small amount of data was collected, 95\% confidence intervals were calculated from the sample. The calculations were based on genealogical data collected for 8 families from the Kingdom of Poland and 9 Lithuanian families.

\section{Keywords}

life expectancy, life tables, aristocracy, magnatery, elites, the Polish-Lithuanian Commonwealth wyników z wartością oczekiwanej długości życia wyliczonej wcześniej dla arystokracji zachodnioeuropejskiej. Dokonano również próby sprawdzenia, czy zachodziły różnice między oczekiwaną długością życia magnatów i magnatek z obu części Rzeczypospolitej: Korony i Wielkiego Księstwa Litewskiego. Ze względu na niewielką liczbę przypadków, na podstawie których dokonano kalkulacji, obliczono też $95 \%$ przedziały ufności.

\section{Słowa kluczowe}

oczekiwana długość życia, tablice trwania życia, arystokracja, magnateria, elity, Rzeczpospolita Obojga Narodów

\section{Introduction}

There is nothing in the world more certain than death, but a man is not sure when his time will come. People pass away from this world: some in old age, some in middle age, some at an immature age; and many of them barely see the light of the sun and at a very young age, for various reasons, they are called upon by God. And this happened with my beloved children, who were a gift from God, and then, much to my great regret, He took them away. Because of that, we have experienced more sorrow from having our children than joy.

The last will of Jerzy Radziwiłł, castellan of Troki (1578-1613). ${ }^{1}$

Life expectancy is one of the demographic parameters closely related to the biological standard of living of individuals and social groups. ${ }^{2}$ Unfortunately,

\footnotetext{
${ }^{1}$ The last will published by Urszula Augustyniak, Testamenty ewangelików reformowanych w Wielkim Księstwie Litewskim w XVI-XVIII wieku (Warszawa: Semper, 2014): 140-148.

${ }^{2}$ The Decline of Infant Mortality in Europe 1800-1950. Four National Case Studies, ed. C.A. Corsini and P.P. Viazzo (Florence: Unicef, 1993); Paul Huck, "Infant Mortality and Living Standards of English Workers During the Industrial Revolution," Journal Of Economic History 55, no. 3 (1995): 528-550; Simon Szreter, Graham Mooney, "Urbanization, Mortality, and the Standard of Living Debate: New Estimates of the Expectation of Life at Birth in Nineteenth-Century British Cities," Economic History Review 51, no. 1 (1998): 84-112; Richard U. Steckel, "Biological Measures of the Standard of Living," Journal of Economic Perspectives 22, no. 1 (2008): 129-152; Jan Luiten Van Zanden et al., How was life? Global Well-Being since 1820 (OECD publishing,
} 
systematic studies on life expectancy in the Polish-Lithuanian Commonwealth in the 16th-18th centuries, have, so far, been concentrated on lower estates, especially peasants. ${ }^{3}$ This was determined, foremost, by the availability of the statistical material - mainly in the form of parish registers and census-like sources. ${ }^{4}$ In terms of the social elite of the Kingdom of Poland and the Grand Duchy of Lithuania, the matter was barely broached, although some research was done during the interwar period. According to Tadeusz Furtak, in the 16th century, average age at death was 59 , in the 17 th century 58.9 , and in the 18 th century $61.8,{ }^{5}$ but his studies were carried out for the nobility and magnatery together, were based on heraldic material of different substantive value and covered a small number of cases. Such an approach means that these calculations are wholly inadequate for any comparison with the aristocratic elites of European countries. The same comments can apply to Egon Vielrose's research. He calculated the average life expectancy to be 50 . In the 16th century it was 18.1 years (102 observations), in the 17th century, 19.4 years (but only on the basis of 70 observations), and in the 18th century, 19.6 years (210 observations). As the author himself noted, these numbers are, however, too high for the entire nobility. ${ }^{6}$

An interesting polemic, well known in Polish historiography, on the biological condition of the Polish-Lithuanian magnatery from the 16th-18th centuries, emerged at the turn of the 1960s and 1970s. It began with an article by Zbigniew Kuchowicz, who referred to the degeneration of the particular magnate houses by the end of the 18th century. ${ }^{7}$ In response to this article, Emanuel Rostworowski calculated the average age of death for adults at the time of death based on the

2014); Richard A. Easterlin, “The Worldwide Standard of Living since 1800," Journal of Economic Perspectives 14, no. 1 (2000): 7-26.

${ }^{3}$ Regarding other social groups or other centuries, it is necessary to mention E. Piasecki's and M. Kędelski's works: Edmund Piasecki, "Próba sporządzenia okresowych tablic życia na podstawie ksiąg ruchu naturalnego w XVIII-XX w.," Przeszłość Demograficzna Polski 16 (1985): 139151; Piasecki, Ludność parafii bejskiej (woj. kieleckie) w świetle ksiag metrykalnych z XVIII-XX w. Studium demograficzne, Wrocław-Warszawa: PWN, 1990), 281-304; Mieczysław Kędelski, "Piramida wieku i tablice trwania życia ludności Śląska w 1864 roku," Przeszłość Demograficzna Polski 20 (1997): 49-71; Kędelski, "Ewolucja umieralności i trwania życia ludności miasta Poznania w wiekach XIX i XX," Studia Demograficzne 83 (1986): 3-26; Kędelski, "Umieralność i trwanie życia w Wielkopolsce w latach 1816-1875," Przeszłość Demograficzna Polski 16 (1985): 109-138; Kędelski, Umieralność i trwanie życia ludności Wielkopolski w XIX wieku (Poznań: Akademia Ekonomiczna w Poznaniu, 1996).

${ }^{4}$ Mikołaj Szołtysek, Rethinking East-Central Europe: Family Systems and Co-Residence in the Polish-Lithuanian Commonwealth (Bern: Peter Lang, 2015): 245-265.

${ }^{5}$ Tadeusz Furtak, "Kilka zagadnień z demografii historycznej szlachty polskiej," Roczniki Dziejów Społecznych i Gospodarczych 4 (1937): 41.

${ }^{6}$ Egon Vielrose, "Przyczynek do demografii szlachty polskiej," Przegląd Statystyczny 1 (1938): 333.

${ }^{7}$ Zbigniew Kuchowicz, "Społeczne konsekwencje postępującej degeneracji możnowładztwa polskiego w XVII-XVIII w."” Kwartalnik Historyczny 76, no. 1 (1969): 21-43; Kuchowicz, 
lifespan of secular and clergy senators in the 16th-18th centuries. ${ }^{8}$ In the first half of the 16th century this came to 52 years, while in the 18th century the average age was approaching 58 years! The author of the article used these results as evidence of the good state of health of Polish magnates. However, from the point of view of demographic analysis, calculating the average age at death does not tell us much about the standard of living or the level of health of the population. Unfortunately, neither Kuchowicz nor Rostworowski calculated life expectancy at the moment of birth (e 0 - very difficult to obtain, as we write below), nor life expectancy at, e.g., the ages of 20 or 50.

The age of adults at the time of death was also discussed in works on other, non-demographic issues ${ }^{9}$ and articles of a demographic nature, but not directly related to life expectancy. ${ }^{10}$ However, research into life expectancy began to be carried out on the Western European aristocracy some decades ago. The topic emerged as an object of research interest for several acclaimed historical demographers such as Sigismund Peller, ${ }^{11}$ Thomas H. Hollingsworth, ${ }^{12}$ Louis Henry,

"Jeszcze o zdrowiu i niezdrowiu magnatów XVII-XVIII w.," Kwartalnik Historyczny 77, no. 2 (1970): 394-403.

${ }^{8}$ Emanuel Rostworowski, "Zdrowie i niezdrowie polskich magnatów XVI-XVIII wieku," Kwartalnik Historyczny 76, no. 4 (1969): 865-886.

${ }^{9}$ Anna Lesiak, Kobiety z rodu Radziwittów w świetle inwentarzy i testamentów (XVIXVIII w.) in Administracja i życie codzienne w dobrach Radziwiltów XVI-XVIII wieku, "Fasciculi Historici Novi," vol. IX, ed. Urszula Augustyniak (Warszawa: Wydawnictwo DiG, 2009): 119130; Raimonda Ragauskienè, Mirties nugaleti nepavyko: Biržu ir Dubingiu Kunigaikščų Radvilu biologine istorija (XV a. pabaiga-XVII a.), (Vilnius: Lietuvos edukologijos universiteto leidykla, 2017) (concerns the Calvinist side of the Radziwiłł family).

${ }^{10}$ Marzena Liedke, "Śmierć magnata, czyli co liczby mogą powiedzieć o konsekwencji śmierci mężczyzny w rodzinie magnackiej w Wielkim Księstwie Litewskim w XVI-XVIII w.," Kwartalnik Historii Kultury Materialnej 60, no. 2 (2012): 271-282 (Radziwiłłs, Ogińskis, Chodkiewiczes).

${ }^{11}$ For articles on mortality and fertility among the members of ruling houses in Europe, see Sigismund Peller, "Studies on Mortality since the Renaissance," Bulletin of the History of Medicine part 1: 13 (1943): 422-461, part 2: 16 (1944): 362-281; Peller, "Mortality, Past and Future," Population Studies 1, no. 4 (1948); Peller, "Births and Deaths among Europe's Ruling Families since 1500," in Population in History. Essays in Historical Demography, eds. David V. Glass and David E.C. Eversley (London: E. Arnold, 1965), 87-101.

${ }^{12}$ Publications of Thomas H. Hollingsworth on the demography of British dukes and the whole peerage (e.g., infant mortality and life expectancy in particular age cohorts of men and women): Thomas Henry Hollingsworth, "A Demographic Study of the British Ducal Families," Population Studies 2, no. 1 (1957): 4-26 (hereafter: A Demographic Study of the British Ducal Families (1)) and reprint in: Hollingsworth, Population in History: Essays in Historical Demography, London: E. Arnold, 1965): 354-378; Hollingsworth, "The Demography of the British Peerage," Population Studies 18, no. 2 (1964) (Supplement): 1-108; Hollingsworth, "Mortality in the British Peerage Families since 1600," Population 32, no. 1 (1977): 323-352. 
Claude Levy, ${ }^{13}$ and, recently, Paul A. David, S. Ryan Johansson, Andrea Pozzi, ${ }^{14}$ Neil Cummins ${ }^{15}$ and, in relation to the life expectancy of members of the Austrian Academy of Sciences, Gustav Feichtinger, Maria Winkler-Dworak, Inga Freund and Alexia Prskawetz. ${ }^{16}$

\section{Aims of the Article, Terminology, Sources and Methods}

As I was aware of the existence of a serious research gap in Polish historiography (but also in Lithuanian, Belarussian or Ukrainian), in 2015, 2016 and 2019', I calculated the life expectancy of magnates (e18, e20, e50). In this article, I have taken my research further by preparing "life tables" (from e20). I also compared the results of my research with the results obtained for the Western European aristocracy.

At this point, it should be noted that the magnatery was not an exact equivalent to the European aristocracy..$^{18}$ Most of its members did not use aristocratic titles. The "Magnatery" consisted of lords who had entered the political elite by the ruler's decision, descendants of ancient genuine knyazes of various origins (in the Grand Duchy of Lithuania) and magnates who did use aristocratic titles but had obtained them from foreign monarchs. Thus, people who had been politically promoted from the middle nobility quite frequently became members of this group. They received high offices and land grants. Therefore, they did not have

${ }^{13}$ Article characterizing demographically the stratum of French dukes and peers (e.g., mortality among men and women, life expectancy of people of a certain age): Louis Henry, Claude Levy, "Ducs et pairs sous l'ancien régime: Caractéristiques démographiques d'une caste," Population 15 (1960): 807-830.

${ }^{14}$ Studies referring to the life expectancy of the members of the British ruling families: Paul A. David, S. Ryan Johansson, Andrea Pozzi, "The Demography of an Early Mortality Transition: Life Expectancy, Survival and Mortality Rates for Britain's Royals, 1500-1799," University of Oxford Discussion Papers in Economic and Social History 83 (2010): 1-59.

${ }^{15}$ Studies raising the problem of life expectancy in the European elites: Neil Cummins, "Lifespans of the European Elite, 800-1800," The Journal of Economic History 77, no. 2 (2017): 406-439.

${ }^{16}$ Gustav Feichtinger, et al., "On the Age Dynamics of Learned Societies - Taking the Example of the Austrian Academy of Sciences," Vienna Yearbook of Population Research, Vienna Institute of Demography, Austrian Academy of Sciences (2007): 107-131.

${ }^{17}$ Marzena Liedke, "Demografia rodziny magnackiej w Wielkim Księstwie Litewskim na tle elit zachodnioeuropejskich. Wybrane problemy," Przeszłość Demograficzna Polski 37, no. 1 (2015): 37-70; Liedke, Rodzina magnacka w Wielkim Księstwie Litewskim. Studium demograficzno-społeczne (Białystok: Instytut Badań nad Dziedzictwem Kulturowym Europy, 2016); Liedke, "Rodzina magnacka" in Rodzina i jej gospodarstwo na ziemiach polskich w geografii europejskich struktur rodzinnych do połowy XIX wieku, eds. P. Guzowski and C. Kuklo (Białystok: Instytut Badań nad Dziedzictwem Kulturowym Europy, 2019), 173-218.

${ }^{18}$ See for example: Jerzy Lukowski, The European Nobility in the Eighteenth Century (New York: Palgrave Macmillan, 2003). 
to be born into this group, as was usual for aristocrats, ${ }^{19}$ at least at the beginning of their lives, e.g., their biological condition could have been different, referring to the condition of the middle nobility. ${ }^{20}$

The magnatery was an informal social group which, nonetheless, had the most important impact on the home and foreign policies of the state, and this social group was, formally, part of a numerous nobility (6-7\% of society) - politically, magnates had the same rights and privileges as common nobles, but it was mainly their representatives that held the highest positions in the state and were members of the Senate (the higher chamber of the parliament of the PolishLithuanian Commonwealth). Their lifestyle was also different from that of nobles. In accordance with the informal name of the group (derived from Latin "magnus"), they were also the economically best-off part of society. Therefore, I have to analyze this group separately from the nobility.

The calculation of the life expectancy parameter values was made on the basis of genealogical facts, such as the birth and death dates of the members of families such as (in the Polish Kingdom): the Leszczyńskis, Opalińskis, Ossolińskis, Ostrogskis/Zasławskis, Lubomirskis, Branickis, Sieniawskis, Zborowskis and (in the Grand Duchy of Lithuania): the Radziwiłłs, Chodkiewiczes, Paces, Ogińskis, Kiszkas, Pociejs, Hlebowiczes, Tyszkiewiczes, Sapiehases (the number of cases: over 365 adult men and 173 adult women; extreme dates of birth for the cohort are, in the case of men: 1457-1800 and women: 1499-1796). The information was scattered over many publications and archival sources. They were acquired foremost from genealogical works and published materials referring to particular families, biographies of particular people, and also biographies published in the Polski słownik biograficzny (Polish Biographical Dictionary), monographs of families, articles dedicated to these families and their members, as well as already-published sources, such as wills, letters and memoirs. Certain data were complemented or corrected as a result of archive query. ${ }^{21}$

${ }^{19}$ Usual, because aristocratic titles could also be obtained.

${ }^{20}$ The biological condition of the middle nobility is considered to be the best among all the social groups of the Polish-Lithuanian Commonwealth - see Zbigniew Kuchowicz, O biologiczny wymiar historii (Łódź: Łódzkie Towarzystwo Naukowe, 1972), 112+.

${ }^{21}$ Foremost: Central Archives of Historical Records in Warsaw (including the Archive of the Radziwiłłs, dz. IV, V, XI), National Archive in Krakow (including the Młynowskie Archive of the Chodkiewiczes), the Lithuanian State Historical Archive in Vilnius (e.g. fond 1177, description 1 referring to the Ogińskis); genealogical works: W. Dworzaczek, Genealogia, vol. 2 (tables), (Warszawa 1959); Józef Wolff, Kniaziowie litewsko-ruscy od końca XIV w. (Warszawa, 1895); Józef Wolff, Pacowie. Materyjały historyczno-genealogiczne, St. Petersburg 1885; Sapiehowie. Materiały historyczno-genealogiczne i majatkowe. Wydane nakładem rodziny, eds. K. Ożarowski and B. Gorczak, vols. 1-3, St. Petersburg 1890-1894; Stanisław Mackiewicz, Dom Radziwittów, Warszawa 1990; Materyaly historyczno-genealogiczne do monografii domu Tyszkiewiczów, ed. M. Tyszkiewicz, vol. 1: wieki XV i XVI (1413-1599), Warszawa 1911; biographies (e. g.,) 
Unfortunately, it was not possible to collect the relevant data on all the family members. ${ }^{22}$ Due to incomplete data regarding children born and those who never reached adulthood, as well as the low number of documented cases of a magnate's birth and death date, it was difficult to calculate the life expectancy at the moment of birth. We have well-documented data for very few families. The example of the Radziwiłłs shows how important they are. In the case of this family, almost half the offspring did not reach adulthood (Table 1).

Table 1. Children born and children who survived to adulthood (the case of the Radziwiłls)

\begin{tabular}{llcc}
\hline Marriages & \multicolumn{1}{c}{ Specification } & Children born & Children surviving to adulthood \\
\hline \multirow{4}{*}{ First } & Total & 115.0 & 63.0 \\
& On average per marriage & 5.4 & 3.0 \\
& Sons & 64.0 & 32.0 \\
& Daughters & 51.0 & 31.0 \\
\hline \multirow{5}{*}{ Subsequent } & Total & 14.0 & 7.0 \\
& On average, per marriage & 4.7 & 2.3 \\
& Sons & 6.0 & 2.0 \\
\hline Total & Daughters & 8.0 & 5.0 \\
\hline
\end{tabular}

Source: Marzena Liedke, database.

Henryk Wisner, Janusz Radziwitt 1612-1655, Warszawa 2000; Tomasz Kempa, Mikołaj Krzysztof Radziwiłt Sierotka (1549-1616). Wojewoda wileński, Warszawa 2000; Marek Ferenc, Mikołaj Radziwiłt Rudy (ok. 1515-1584). Działalność polityczna i wojskowa, Kraków 2008; memoirs (including) Genealogia imienia naszego [Genealogia Imienia Pociejów] wypisana z Manuskryptów Jaśnie Wielmożnego Pana Ludwika Kon: na Włodawie i Rożanie Pocieja Wojewody Wileńskiego Hetmana Wgo Xa Litewskiego, Szerszowskeigo, Puńskiego, Ratyńskiego, Stolińskiego etc. Starosty, "Przegląd Poznański" 30, 1860, 210-228; Albrycht Stanisław Radziwiłł, Pamiętnik o dziejach w Polsce vol. 1 (1632-1636), vol. 2 (1637-1646), vol. 3 (1647-1656), published by A. Przyboś, R, Żelewski, Warszawa 1980; B. Radziwiłł, Autobiografia, ed. T. Wasilewski, Warszawa 1979; Hieronima Floriana Radziwiłta Diariusze i pisma różne, ed. and introduced by M. Brzezina, Warszawa 1998; Marcin Matuszewicz, Diariusz życia mego, ed. B. Królikowski, vol. 1 (1714-1757),Warszawa 1986; Marcin Matuszewicz, Diariusz życia mego, vol. 2, ed. B. Królikowski (1758-1764),Warszawa 1986 and, e.g., unpublished, Michał Kazimierz, Radziwiłł's diary (Archiwum Główne Akt Dawnych w Warszawie, Archiwum Radziwiłłów, dział VI, sygn. II-80 a). For a list of literature and manuscript sources see also: Marzena Liedke, "Demografia rodziny magnackiej," 41-42.

${ }^{22}$ Such difficulties refer not only to Polish and Lithuanian works, but also those from Western Europe. S. Peller, dealing with the demography of European reigning families, also indicated incompleteness in the data; for instance, in the 16th century, in the genealogical tables of the family of Isenburg, the information on the age at the moment of marriage of $6 \%$ men and $29 \%$ women is missing: Sigismund Peller, "Mortality, Past and Future," 405. 
It was impossible to examine the e 0 parameter, but we managed to calculate the life expectancy, in order to facilitate comparisons with Western European elites, at the ages of 20 and 50. Life tables were also constructed starting from the age of 20 (Tables 2 and 3). On account of the relatively small number of cases, the entire group was adopted as the cohort constituting the basis for constructing the life tables.

Table 2. Life tables of magnate males in the Polish-Lithuanian Commonwealth in the early modern period

\begin{tabular}{|c|c|c|c|c|c|c|c|}
\hline \multirow{2}{*}{ Age } & \multicolumn{7}{|c|}{ Parameters } \\
\hline & $\mathrm{dx}$ & $1 \mathrm{x}$ & $q x$ & Lx & $m x$ & $\mathrm{Tx}$ & ex \\
\hline 1 & 2 & 3 & 4 & 5 & 6 & 7 & 8 \\
\hline 20 & 2 & 365 & 0.00547945 & 364.0 & 0.00549451 & $11,971.5$ & 32.80 \\
\hline 21 & 3 & 363 & 0.00826446 & 361.5 & 0.00829876 & $11,607.5$ & 31.98 \\
\hline 22 & 3 & 360 & 0.00833333 & 358.5 & 0.00836820 & $11,246.0$ & 31.24 \\
\hline 23 & 4 & 357 & 0.01120448 & 355.0 & 0.01126761 & $10,887.5$ & 30.50 \\
\hline 24 & 2 & 353 & 0.00566572 & 352.0 & 0.00568182 & $10,532.5$ & 29.84 \\
\hline 25 & 3 & 351 & 0.00854701 & 349.5 & 0.00858369 & $10,180.5$ & 29.00 \\
\hline 26 & 5 & 348 & 0.01436782 & 345.5 & 0.01447178 & $9,831.0$ & 28.25 \\
\hline 27 & 8 & 343 & 0.02332362 & 339.0 & 0.02359882 & $9,485.5$ & 27.65 \\
\hline 28 & 2 & 335 & 0.00597015 & 334.0 & 0.00598802 & $9,146.5$ & 27.30 \\
\hline 29 & 6 & 333 & 0.01801802 & 330.0 & 0.01818182 & $8,812.5$ & 26.46 \\
\hline 30 & 6 & 327 & 0.01834862 & 324.0 & 0.01851852 & $8,482.5$ & 25.94 \\
\hline 31 & 3 & 321 & 0.00934579 & 319.5 & 0.00938967 & $8,158.5$ & 25.42 \\
\hline 32 & 4 & 318 & 0.01257862 & 316.0 & 0.01265823 & $7,839.0$ & 24.65 \\
\hline 33 & 6 & 314 & 0.01910828 & 311.0 & 0.01929261 & $7,523.0$ & 23.96 \\
\hline 34 & 4 & 308 & 0.01298701 & 306.0 & 0.01307190 & $7,212.0$ & 23.42 \\
\hline 35 & 5 & 304 & 0.01644737 & 301.5 & 0.01658375 & $6,906.0$ & 22.72 \\
\hline 36 & 3 & 299 & 0.01003345 & 297.5 & 0.01008403 & $6,604.5$ & 22.09 \\
\hline 37 & 7 & 296 & 0.02364865 & 292.5 & 0.02393162 & $6,307.0$ & 21.31 \\
\hline 38 & 7 & 289 & 0.02422145 & 285.5 & 0.02451839 & $6,014.5$ & 20.81 \\
\hline 39 & 5 & 282 & 0.01773050 & 279.5 & 0.01788909 & $5,729.0$ & 20.32 \\
\hline 40 & 5 & 277 & 0.01805054 & 274.5 & 0.01821494 & $5,449.5$ & 19.67 \\
\hline 41 & 6 & 272 & 0.02205882 & 269.0 & 0.02230483 & $5,175.0$ & 19.03 \\
\hline 42 & 6 & 266 & 0.02255639 & 263.0 & 0.02281369 & $4,906.0$ & 18.44 \\
\hline 43 & 6 & 260 & 0.02307692 & 257.0 & 0.02334630 & $4,643.0$ & 17.86 \\
\hline 44 & 7 & 254 & 0.02755906 & 250.5 & 0.02794411 & $4,386.0$ & 17.27 \\
\hline 45 & 7 & 247 & 0.02834008 & 243.5 & 0.02874743 & $4,135.5$ & 16.74 \\
\hline 46 & 10 & 240 & 0.04166667 & 235.0 & 0.04255319 & $3,892.0$ & 16.22 \\
\hline 47 & 10 & 230 & 0.04347826 & 225.0 & 0.04444444 & $3,657.0$ & 15.90 \\
\hline 48 & 6 & 220 & 0.02727273 & 217.0 & 0.02764977 & $3,432.0$ & 15.60 \\
\hline 49 & 7 & 214 & 0.03271028 & 210.5 & 0.03325416 & $3,215.0$ & 15.02 \\
\hline 50 & 8 & 207 & 0.03864734 & 203.0 & 0.03940887 & $3,004.5$ & 14.51 \\
\hline
\end{tabular}




\begin{tabular}{|c|c|c|c|c|c|c|c|}
\hline 1 & 2 & 3 & 4 & 5 & 6 & 7 & 8 \\
\hline 51 & 5 & 199 & 0.02512563 & 196.5 & 0.02544529 & $2,801.5$ & 14.08 \\
\hline 52 & 3 & 194 & 0.01546392 & 192.5 & 0.01558442 & $2,605.0$ & 13.43 \\
\hline 53 & 9 & 191 & 0.04712042 & 186.5 & 0.04825737 & $2,412.5$ & 12.63 \\
\hline 54 & 7 & 182 & 0.03846154 & 178.5 & 0.03921569 & $2,226.0$ & 12.23 \\
\hline 55 & 7 & 175 & 0.04000000 & 171.5 & 0.04081633 & $2,047.5$ & 11.70 \\
\hline 56 & 8 & 168 & 0.04761905 & 164.0 & 0.04878049 & $1,876.0$ & 11.17 \\
\hline 57 & 9 & 160 & 0.05625000 & 155.5 & 0.05787781 & $1,712.0$ & 10.70 \\
\hline 58 & 10 & 151 & 0.06622517 & 146.0 & 0.06849315 & $1,556.5$ & 10.31 \\
\hline 59 & 7 & 141 & 0.04964539 & 137.5 & 0.05090909 & $1,410.5$ & 10.00 \\
\hline 60 & 12 & 134 & 0.08955224 & 128.0 & 0.09375000 & $1,273.0$ & 9.50 \\
\hline 61 & 8 & 122 & 0.06557377 & 118.0 & 0.06779661 & $1,145.0$ & 9.39 \\
\hline 62 & 8 & 114 & 0.07017544 & 110.0 & 0.07272727 & $1,027.0$ & 9.01 \\
\hline 63 & 14 & 106 & 0.13207547 & 99.0 & 0.14141414 & 917.0 & 8.65 \\
\hline 64 & 6 & 92 & 0.06521739 & 89.0 & 0.06741573 & 818.0 & 8.89 \\
\hline 65 & 6 & 86 & 0.06976744 & 83.0 & 0.07228916 & 729.0 & 8.48 \\
\hline 66 & 10 & 80 & 0.12500000 & 75.0 & 0.13333333 & 646.0 & 8.08 \\
\hline 67 & 6 & 70 & 0.08571429 & 67.0 & 0.08955224 & 571.0 & 8.16 \\
\hline 68 & 3 & 64 & 0.04687500 & 62.5 & 0.04800000 & 504.0 & 7.88 \\
\hline 69 & 4 & 61 & 0.06557377 & 59.0 & 0.06779661 & 441.5 & 7.24 \\
\hline 70 & 8 & 57 & 0.14035088 & 53.0 & 0.15094340 & 382.5 & 6.71 \\
\hline 71 & 3 & 49 & 0.06122449 & 47.5 & 0.06315790 & 329.5 & 6.72 \\
\hline 72 & 6 & 46 & 0.13043478 & 43.0 & 0.13953488 & 282.0 & 6.13 \\
\hline 73 & 7 & 40 & 0.17500000 & 36.5 & 0.19178082 & 239.0 & 5.98 \\
\hline 74 & 5 & 33 & 0.15151515 & 30.5 & 0.16393443 & 202.5 & 6.14 \\
\hline 75 & 1 & 28 & 0.03571429 & 27.5 & 0.03636364 & 172.0 & 6.14 \\
\hline 76 & 3 & 27 & 0.11111111 & 25.5 & 0.11764706 & 144.5 & 5.35 \\
\hline 77 & 4 & 24 & 0.16666667 & 22.0 & 0.18181818 & 119.0 & 4.96 \\
\hline 78 & 6 & 20 & 0.30000000 & 17.0 & 0.35294118 & 97.0 & 4.85 \\
\hline 79 & 1 & 14 & 0.07142857 & 13.5 & 0.07407407 & 80.0 & 5.71 \\
\hline 80 & 3 & 13 & 0.23076923 & 11.5 & 0.26086957 & 66.5 & 5.12 \\
\hline 81 & 2 & 10 & 0.20000000 & 9.0 & 0.22222222 & 55.0 & 5.50 \\
\hline 82 & 2 & 8 & 0.25000000 & 7.0 & 0.28571429 & 46.0 & 5.75 \\
\hline 83 & 0 & 6 & 0.00000000 & 6.0 & 0.00000000 & 39.0 & 6.50 \\
\hline 84 & 0 & 6 & 0.00000000 & 6.0 & 0.00000000 & 33.0 & 5.50 \\
\hline 85 & 1 & 6 & 0.16666667 & 5.5 & 0.18181818 & 27.0 & 4.50 \\
\hline 86 & 0 & 5 & 0.00000000 & 5.0 & 0.00000000 & 21.5 & 4.30 \\
\hline 87 & 1 & 5 & 0.20000000 & 4.5 & 0.22222222 & 16.5 & 3.30 \\
\hline 88 & 0 & 4 & 0.00000000 & 4.0 & 0.00000000 & 12.0 & 3.00 \\
\hline 89 & 2 & 4 & 0.50000000 & 3.0 & 0.666666667 & 8.0 & 2.00 \\
\hline 90 & 0 & 2 & 0.00000000 & 2.0 & 0.00000000 & 5.0 & 2.50 \\
\hline 91 & 0 & 2 & 0.00000000 & 2.0 & 0.00000000 & 3.0 & 1.50 \\
\hline 92 & 2 & 2 & 1.00000000 & 1.0 & 2.00000000 & 1.0 & 0.50 \\
\hline
\end{tabular}

Source: Marzena Liedke, database. 
Table 3. Life tables of magnate women in the Polish-Lithuanian Commonwealth in the early modern period

\begin{tabular}{|c|c|c|c|c|c|c|c|}
\hline \multirow{2}{*}{ Age } & \multicolumn{7}{|c|}{ Parameters } \\
\hline & $\mathrm{dx}$ & $1 \mathrm{x}$ & $\mathrm{qx}$ & $\mathrm{Lx}$ & $\mathrm{mx}$ & $\mathrm{Tx}$ & ex \\
\hline 1 & 2 & 3 & 4 & 5 & 6 & 7 & 8 \\
\hline 20 & 3 & 169 & 0.01775148 & 167.5 & 0.01791045 & $5,257.5$ & 31.11 \\
\hline 21 & 3 & 166 & 0.01807229 & 164.5 & 0.01823708 & $5,090.0$ & 30.66 \\
\hline 22 & 0 & 163 & 0.00000000 & 163.0 & 0.00000000 & $4,925.5$ & 30.22 \\
\hline 23 & 1 & 163 & 0.00613497 & 162.5 & 0.00615385 & $4,762.5$ & 29.22 \\
\hline 24 & 5 & 162 & 0.03086420 & 159.5 & 0.03134796 & $4,600.0$ & 28.40 \\
\hline 25 & 1 & 157 & 0.00636943 & 156.5 & 0.00638978 & $4,440.5$ & 28.28 \\
\hline 26 & 3 & 156 & 0.01923077 & 154.5 & 0.01941748 & $4,284.0$ & 27.46 \\
\hline 27 & 9 & 153 & 0.05882353 & 148.5 & 0.06060606 & $4,129.5$ & 26.99 \\
\hline 28 & 4 & 144 & 0.02777778 & 142.0 & 0.02816901 & $3,981.0$ & 27.65 \\
\hline 29 & 2 & 140 & 0.01428571 & 139.0 & 0.01438849 & $3,839.0$ & 27.42 \\
\hline 30 & 2 & 138 & 0.01449275 & 137.0 & 0.01459854 & $3,700.0$ & 26.81 \\
\hline 31 & 2 & 136 & 0.01470588 & 135.0 & 0.01481482 & $3,563.0$ & 26.20 \\
\hline 32 & 3 & 134 & 0.02238806 & 132.5 & 0.02264151 & $3,428.0$ & 25.58 \\
\hline 33 & 3 & 131 & 0.02290076 & 129.5 & 0.02316602 & $3,295.5$ & 25.16 \\
\hline 34 & 2 & 128 & 0.01562500 & 127.0 & 0.01574803 & $3,166.0$ & 24.73 \\
\hline 35 & 4 & 126 & 0.03174603 & 124.0 & 0.03225807 & $3,039.0$ & 24.12 \\
\hline 36 & 1 & 122 & 0.00819672 & 121.5 & 0.00823045 & $2,915.0$ & 23.89 \\
\hline 37 & 4 & 121 & 0.03305785 & 119.0 & 0.03361345 & $2,793.5$ & 23.09 \\
\hline 38 & 5 & 117 & 0.04273504 & 114.5 & 0.04366812 & $2,674.5$ & 22.86 \\
\hline 39 & 4 & 112 & 0.03571429 & 110.0 & 0.03636364 & $2,560.0$ & 22.86 \\
\hline 40 & 3 & 108 & 0.02777778 & 106.5 & 0.02816901 & $2,450.0$ & 22.69 \\
\hline 41 & 4 & 105 & 0.03809524 & 103.0 & 0.03883495 & $2,343.5$ & 22.32 \\
\hline 42 & 4 & 101 & 0.03960396 & 99.0 & 0.04040404 & $2,240.5$ & 22.18 \\
\hline 43 & 0 & 97 & 0.00000000 & 97.0 & 0.00000000 & $2,141.5$ & 22.08 \\
\hline 44 & 5 & 97 & 0.05154639 & 94.5 & 0.05291005 & $2,044.5$ & 21.08 \\
\hline 45 & 1 & 92 & 0.01086957 & 91.5 & 0.01092896 & $1,950.0$ & 21.20 \\
\hline 46 & 1 & 91 & 0.01098901 & 90.5 & 0.01104972 & $1,858.5$ & 20.42 \\
\hline 47 & 3 & 90 & 0.03333333 & 88.5 & 0.03389831 & $1,768.0$ & 19.64 \\
\hline 48 & 4 & 87 & 0.04597701 & 85.0 & 0.04705882 & $1,679.5$ & 19.30 \\
\hline 49 & 3 & 83 & 0.03614458 & 81.5 & 0.03680982 & $1,594.5$ & 19.21 \\
\hline 50 & 4 & 80 & 0.05000000 & 78.0 & 0.05128205 & $1,513.0$ & 18.91 \\
\hline 51 & 0 & 76 & 0.00000000 & 76.0 & 0.00000000 & $1,435.0$ & 18.88 \\
\hline 52 & 1 & 76 & 0.01315790 & 75.5 & 0.01324503 & $1,359.0$ & 17.88 \\
\hline 53 & 2 & 75 & 0.02666667 & 74.0 & 0.02702703 & $1,283.5$ & 17.11 \\
\hline 54 & 2 & 73 & 0.02739726 & 72.0 & 0.02777778 & $1,209.5$ & 16.57 \\
\hline 55 & 2 & 71 & 0.02816901 & 70.0 & 0.02857143 & $1,137.5$ & 16.02 \\
\hline 56 & 3 & 69 & 0.04347826 & 67.5 & 0.04444444 & $1,067.5$ & 15.47 \\
\hline 57 & 0 & 66 & 0.00000000 & 66.0 & 0.00000000 & $1,000.0$ & 15.15 \\
\hline 58 & 1 & 66 & 0.01515152 & 65.5 & 0.01526718 & 934.0 & 14.15 \\
\hline
\end{tabular}




\begin{tabular}{rrrrrrrc}
\hline 1 & 2 & 3 & 4 & 5 & 6 & 7 & 8 \\
\hline 59 & 3 & 65 & 0.04615385 & 63.5 & 0.04724409 & 868.5 & 13.36 \\
60 & 6 & 62 & 0.09677419 & 59.0 & 0.10169492 & 805.0 & 12.98 \\
61 & 1 & 56 & 0.01785714 & 55.5 & 0.01801802 & 746.0 & 13.32 \\
62 & 1 & 55 & 0.01818182 & 54.5 & 0.01834862 & 690.5 & 12.55 \\
63 & 2 & 54 & 0.03703704 & 53.0 & 0.03773585 & 636.0 & 11.78 \\
64 & 1 & 52 & 0.01923077 & 51.5 & 0.01941748 & 583.0 & 11.21 \\
65 & 2 & 51 & 0.03921569 & 50.0 & 0.04000000 & 531.5 & 10.42 \\
66 & 4 & 49 & 0.08163265 & 47.0 & 0.08510638 & 481.5 & 9.83 \\
67 & 2 & 45 & 0.04444444 & 44.0 & 0.04545455 & 434.5 & 9.66 \\
68 & 6 & 43 & 0.13953488 & 40.0 & 0.15000000 & 390.5 & 9.08 \\
69 & 0 & 37 & 0.00000000 & 37.0 & 0.00000000 & 350.5 & 9.47 \\
70 & 2 & 37 & 0.05405405 & 36.0 & 0.05555556 & 313.5 & 8.47 \\
71 & 5 & 35 & 0.14285714 & 32.5 & 0.15384615 & 277.5 & 7.93 \\
72 & 2 & 30 & 0.06666667 & 29.0 & 0.06896552 & 245.0 & 8.17 \\
73 & 6 & 28 & 0.21428571 & 25.0 & 0.24000000 & 216.0 & 7.71 \\
74 & 2 & 22 & 0.09090909 & 21.0 & 0.09523810 & 191.0 & 8.68 \\
75 & 1 & 20 & 0.05000000 & 19.5 & 0.05128205 & 170.0 & 8.50 \\
76 & 1 & 19 & 0.05263158 & 18.5 & 0.05405405 & 150.5 & 7.92 \\
77 & 0 & 18 & 0.00000000 & 18.0 & 0.00000000 & 132.0 & 7.33 \\
78 & 0 & 18 & 0.00000000 & 18.0 & 0.00000000 & 114.0 & 6.33 \\
79 & 2 & 18 & 0.11111111 & 17.0 & 0.11764706 & 96.0 & 5.33 \\
80 & 4 & 16 & 0.25000000 & 14.0 & 0.28571429 & 79.0 & 4.94 \\
81 & 1 & 12 & 0.08333333 & 11.5 & 0.08695652 & 65.0 & 5.42 \\
82 & 1 & 11 & 0.09090909 & 10.5 & 0.09523810 & 53.5 & 4.86 \\
83 & 1 & 10 & 0.10000000 & 9.5 & 0.10526316 & 43.0 & 4.30 \\
84 & 2 & 9 & 0.22222222 & 8.0 & 0.25000000 & 33.5 & 3.72 \\
85 & 2 & 7 & 0.28571429 & 6.0 & 0.33333333 & 25.5 & 3.64 \\
86 & 0 & 5 & 0.00000000 & 5.0 & 0.00000000 & 19.5 & 3.90 \\
87 & 1 & 5 & 0.20000000 & 4.5 & 0.22222222 & 14.5 & 2.90 \\
88 & 2 & 4 & 0.50000000 & 3.0 & 0.66666667 & 10.0 & 2.50 \\
89 & 0 & 2 & 0.00000000 & 2.0 & 0.00000000 & 7.0 & 3.50 \\
90 & 0 & 2 & 0.00000000 & 2.0 & 0.00000000 & 5.0 & 2.50 \\
91 & 0 & 2 & 0.00000000 & 2.0 & 0.00000000 & 3.0 & 1.50 \\
92 & 2 & 2 & 1.00000000 & 1.0 & 2.00000000 & 1.0 & 0.50 \\
\hline & & 2 & & & & &
\end{tabular}

Source: Marzena Liedke, database.

As we can see, over the 16th-18th centuries, men's life expectancy at the age of 20 was almost 33 years, while at the age of 50 it was 14.5. On average, women who reached their $20 \mathrm{~s}$ lived a further 31 years, and on reaching the age of 50 they still had 19 years to go.

It might be expected that the life expectancy of women would also be higher at the age of 20, but it should be noted that high maternal mortality had a significant 
Figure 1. Life expectancy for men and women in the Polish-Lithuanian Commonwealth (16th-18th centuries)

- Males .... Females

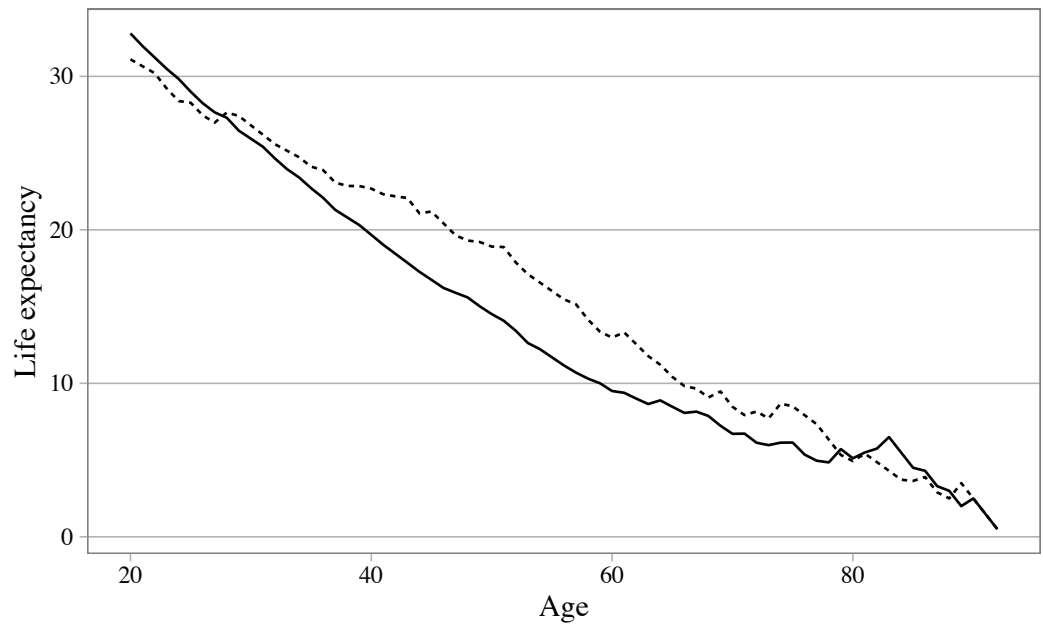

Source: Marzena Liedke, database.

Figure 2. Age at death of magnates in the Polish-Lithuanian Commonwealth (16th-18th centuries)

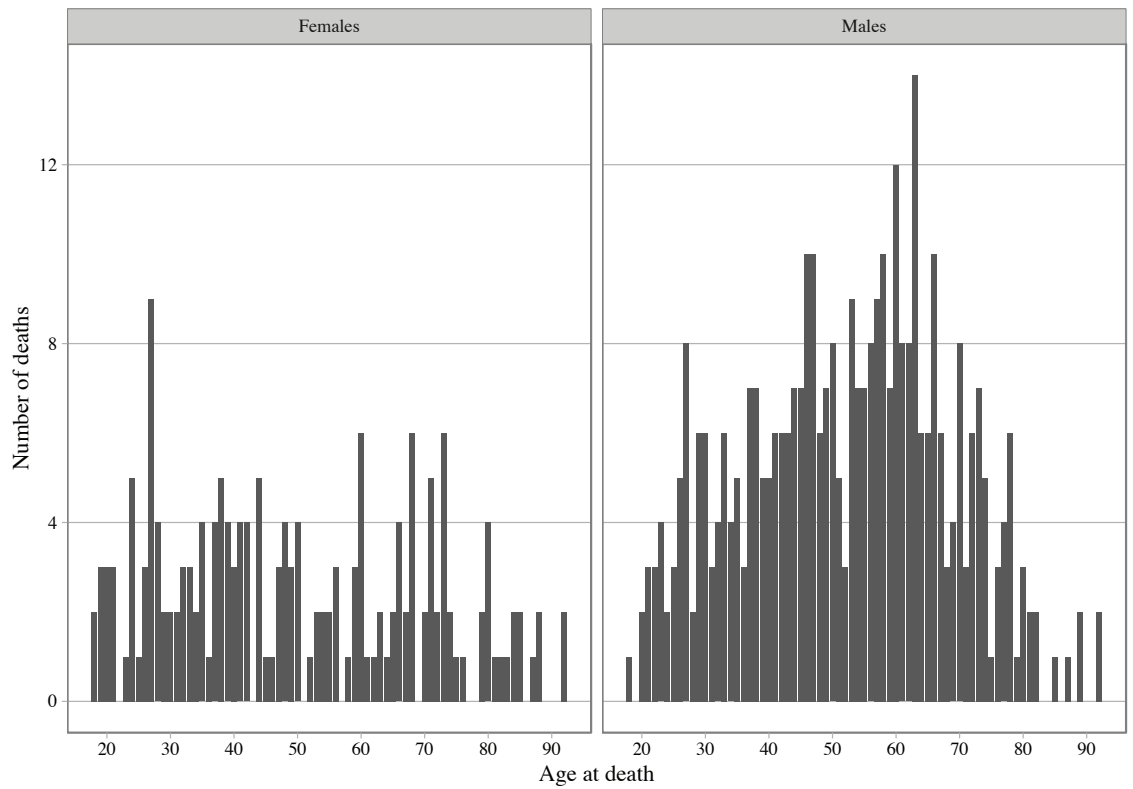

Source: Marzena Liedke, database. 
impact on the value of this parameter (the value of men's life expectancy increases where they reach 30 years of age) and the calculations were made on a much smaller number of cases (poor data recording for women in sources) (Figures 1 and 2).

I also decided to find out if there were differences in life expectancy between men and women from different parts of the Commonwealth (Figure 3). These differences turned out to be minor.

Figure 3. Life expectancy of men and women in KP and GDL (16th-18th centuries)

- Males -... Females

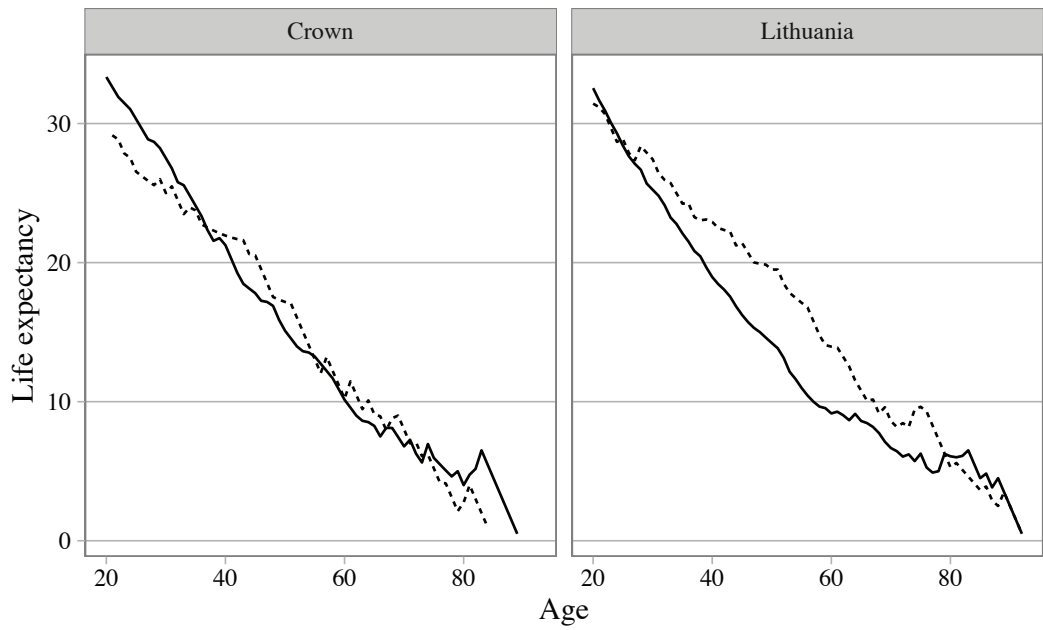

Source: Marzena Liedke, database.

The slight differences that may be observed between the life expectancy of magnates from the Kingdom of Poland and the Grand Duchy of Lithuania may result from the small amount of data collected. Therefore, I decided to calculate $95 \%$ confidence intervals from my sample (Tables 4 and 5 ). ${ }^{23}$

${ }^{23}$ Confidence intervals are based on 1,000 Monte Carlo simulations. They were performed according to the procedure suggested by Evgeny M. Andreev and Vladimir M. Shkolnikov, Spreadsheet for Calculation of Confidence Limits for Any Life Table or Healthy-Life Table Quantity (Max Planck Institute for Demographic Research Technical Report 2010-005, June 2010). 
Table 4. Values of lower bound and upper bound confidence intervals for magnate males in the Grand Duchy of Lithuania and the Kingdom of Poland

\begin{tabular}{ccccccc}
\hline \multicolumn{9}{c}{ Grand Duchy of Lithuania } & \multicolumn{3}{c}{ Kingdom of Poland } \\
\hline Age & Ex & lower bound & upper bound & Ex & lower bound & upper bound \\
\hline 20 & 33.2 & 31.3 & 35.4 & 34.1 & 31.3 & 37.6 \\
30 & 26.4 & 24.6 & 26.5 & 28.3 & 25.8 & 31.5 \\
40 & 20.2 & 18.6 & 22.2 & 21.9 & 19.6 & 24.9 \\
50 & 15.7 & 14.1 & 17.8 & 16.3 & 14.1 & 18.8 \\
60 & 11.5 & 9.8 & 13.9 & 11.5 & 9.5 & 14.0 \\
70 & 8.9 & 7.2 & 12.0 & 8.9 & 6.5 & 11.9 \\
\hline
\end{tabular}

Source: Marzena Liedke, database.

Table 5. Values of lower bound and upper bound confidence intervals for magnate females in the Grand Duchy of Lithuania and the Kingdom of Poland

\begin{tabular}{ccccccc}
\hline \multicolumn{4}{c}{ The Grand Duchy of Lithuania } & \multicolumn{3}{c}{ The Kingdom of Poland } \\
\hline Age & Ex & lower bound & upper bound & Ex & lower bound & upper bound \\
\hline 20 & 32.7 & 29.3 & 36.2 & 31.0 & 25.6 & 36.9 \\
30 & 27.9 & 24.6 & 31.7 & 27.3 & 22.6 & 33.7 \\
40 & 24.0 & 20.9 & 27.5 & 23.8 & 19.4 & 28.9 \\
50 & 20.9 & 18.2 & 24.3 & 16.5 & 13.2 & 21.6 \\
60 & 15.7 & 13.2 & 18.5 & 12.7 & 9.7 & 17.2 \\
70 & 10.1 & 7.6 & 13.4 & 8.1 & 5.8 & 11.7 \\
\hline
\end{tabular}

Source: Marzena Liedke, database.

The differences are not statistically significant. Magnates living both in the Kingdom of Poland and in the Grand Duchy of Lithuania led a similar lifestyle, under very similar economic and environmental conditions. In addition, they were related to each other as a result of marriage between representatives of both states in the Polish-Lithuanian Commonwealth.

However, we can see bigger differences in relation to the Western European elites. Studies conducted by S. Peller, T.H. Hollingsworth and L. Henry and C. Levy referred to various cohorts and time periods, but the longer life expectancy for both men and women is clearly discernible, especially in the second half of the 17th and 18th centuries (Tables 6 and 7.).

T.H. Hollingsworth calculated the value of e15 and e 50 to compare the results with S. Peller's calculations referring to members of ruling families in Europe. ${ }^{24}$ If, for the people who were born in the period 1480-1679 (both women and men),

${ }^{24}$ Thomas H. Hollingsworth, “A Demographic Study of the British Ducal Families,” (1), 12. 
the rate was more favorable for the continental ruling houses, for the cohorts 1680-1779 and 1780-1879 the reverse was true, in the case of life expectancy at the ages of both 15 and 50. In the first case, in the years 1680-1779 a duke's fifteen-year-old son lived on average 37.8 years, while in European families a year less. For dukes born in the years 1780-1879, life expectancy was 45 years, whereas for members of ruling houses in Europe it was 43.7. On the other hand, British ducal sons born in the period 1680-1779 who turned 50, still lived for, on average, 19.3 years, whereas in the continent they lived almost three years shorter. Within the following hundred years, the life expectancy of fifty-year-old members of the British elites was already another 20.5 years, while that of the representatives of European ruling families at the same age was nearly two years shorter. ${ }^{25}$ The results of the 2010 studies on the life expectancy of members of the British royal families in the early modern period demonstrated that the life expectancy of men born in the 16th century was only 18.5, while it grew for those who were born in the period 1550-1649 to 34.8, and remained at that level for those born over the next hundred years (1650-1749), at 34.4 years. People born in the 18th century lived, on average, up to 47.6 years. ${ }^{26}$ Thus, we may conclude that the results are not basically different from the data obtained by T.H. Hollingsworth for British ducal families for the last two decades of the 17th century and the first quarter of the 18th century (e0: c. 33), as well as showing the lengthening tendency for the cohort of the 18th century ( $\mathrm{e} 0$ in the case of people born in the years 1730-79: c. 45 years). In France in the years 1640-1739, the further life expectancy of dukes and peers who turned 20 was 34.5 years. ${ }^{27}$

In the case of women's life expectancy at 15, in British ducal families, daughters born in the years 1680-1779 lived for 41.6 years, while daughters and wives in ruling families in Europe only half as long. In the subsequent period, British fifteen-year-olds still reached nearly 50 (49.6) and the representatives of the continental Europe 45.6. Fifty-year-olds in British families in the cohort 1680-1779 on average died after 21.5 years, while women in the continent after nearly 18 years. Over the next century the former still lived for 24 years (24.2) whereas the latter for $21 .{ }^{28}$ The studies of P.A. David, S.R. Johansson and A. Pozzi in 2010 on female members of British royal families showed that women born in the 16th century, at the moment of birth, lived on average 31 years, and those in the cohort 1550-1649, on average 27 , whereas those born in the 17 th century only lived to be 23 . Rate $\mathrm{e} 0$ grew for the women who were born in the years $1650-1749$, again to 31 years,

\footnotetext{
${ }^{25}$ Thomas H. Hollingsworth, "A Demographic Study of the British Ducal Families” (1), 12.

${ }^{26}$ Paul A. David, S. Ryan Johansson, and Andrea Pozzi, "The Demography of an Early Mortality Transition," 57.

${ }^{27}$ Louis Henry, Claude Levy, "Ducs et pairs," 820.

${ }^{28}$ Thomas H. Hollingsworth, "A Demographic Study of the British Ducal Families," (1), 12.
} 
and up to over 51 for the cohort $1700-99 .{ }^{29}$ On the other hand, the life expectancy of French dukes and peers' daughters who turned 20 was, in the case of those born in the period $1640-1739,33.6$ years. ${ }^{30}$

In the light of these data, the average lifespan of magnate daughters and wives was lower than in European female aristocrats. This may be explained by the inclusion of a lower number of women than men in the studies. Besides, for both men and women from the PLC, the standard of medical care seemed worse than in Western Europe (even though they had better access to services of physicians than other groups of the population). The magnates themselves complained about the questionable skills of doctors (e.g., Bogusław Radziwiłł or Krzysztof Opaliński). ${ }^{31}$ Incorrect diagnoses and inappropriate therapies sometimes caused a worsening of the disease and even the patient's death. Even if the knowledge and experience of doctors were considered satisfactory, it should be recalled that in the case of a standard visit, the doctor made a diagnosis based on observation of the patient, interview (often by letter!) and, possibly, an examination of the patient's discharges, and hardly any physical contact (except for taking the pulse). ${ }^{32}$ Similar remarks can be made for the medicines, which often made the patient's condition worse. However, it is difficult to find studies that would allow a clear comparison of the state of medical care in Western Europe and the Polish-Lithuanian Commonwealth, but studies/research on human capital (which could also reflect health awareness and medical knowledge) indicate that in Eastern Europe, the social level of this resource among the elite was even worse..$^{33}$

Furthermore, the results obtained for women could have been influenced by, for example, relatively high perinatal mortality. It was not until the second half of the 18th century that the idea of taking care of women during pregnancy and the puerperium (e.g., assisting a male doctor in childbirth or the use of forceps)

${ }^{29}$ Paul A. David, S. Ryan Johansson, and Andrea Pozzi, "The Demography of an Early Mortality Transition," 57.

${ }^{30}$ Louis Henry and Claude Levy, "Ducs et pairs," 826.

${ }^{31}$ K. Stojek-Sawicka, "Rola i miejsce lekarzy w życiu elit politycznych Rzeczypospolitej w epoce staropolskiej," in Relacje lekarz-pacjent w aspekcie spolecznym, historycznym i kulturowym, ed. B. Płonka-Syroka (series: Studia z Dziejów Kultury Medycznej, vol. 10), (Warszawa: Wydawnictwo DiG, Oficyna Wydawnicza Arboretum 2005), 127+.

${ }^{32}$ Except for surgeons, of course - Jakub Węglorz, Zdrowie, choroba i lecznictwo w społeczeństwie Rzeczypospolitej XVI-XVIII wieku (Toruń: Wydawnictwo Adam Marszałek, 2015), 82.

${ }^{33}$ See Joerg Baten and Tom Keywood, "Elite Violence and Elite Numeracy in Europe from 500 to 1,900 CE: A Co-Evolution?" (2019), Discussion Papers from C.E.P.R. No. 14013. The situation was similar with regard to the lower social strata - see: Joerg Baten, Mikołaj Szołtysek, and Monika Campestrini “'Girl Power' in Eastern Europe? The Human Capital Development of Central-Eastern Europe in the Seventeenth to Nineteenth Centuries and its Determinants," European Review of Economic History 21, no. 1 (2017): 29-63. 
first began to spread to England, then to Austria, then to other countries in Western Europe. ${ }^{34}$ Judith Lewis's studies on perinatal mortality among British female aristocrats over the 16th-19th centuries indicate that, in that period, only approximately $4.8 \%$ of women died in, or as a result of, childbirth. ${ }^{35}$ The highest percentage of deaths in childbirth was noted, however, for female aristocrats born in the years $1750-74(9.2 \%)$. For the remaining cohorts, by the end of the $18^{\text {th }}$ century, the percentage of deaths related to childbirth vacillated from $7.6 \%$ (1600-24) to 1.4 (1675-99), and on average hovered around $6 \%{ }^{36}$ In the Radziwiłł family, of the 85 wives $\left(16^{\text {th }}-18^{\text {th }}\right.$ centuries), only 60 had children. Of these 60 wives, as many as 7 (about 12\%) died due to perinatal complications; thus, it was, in any case, higher than the rate for British aristocrats.

Table 6. Life expectancy at 15 and 50 in British ducal families and European ruling families (16th-19th centuries)

\begin{tabular}{ccccccccc}
\hline \multirow{2}{*}{$\begin{array}{c}\text { Cohort } \\
\text { of those born }\end{array}$} & \multicolumn{3}{c}{ British ducal families } & \multicolumn{3}{c}{ European ruling families } \\
\cline { 2 - 9 } in the years & \multicolumn{2}{c}{ men } & \multicolumn{2}{c}{ women } & \multicolumn{2}{c}{ men } & \multicolumn{2}{c}{ women } \\
\hline & at 15 & at 50 & at 15 & at 50 & at 15 & at 50 & at 15 & at 50 \\
\hline $1480-1679$ & 27.7 & 15.0 & 33.2 & 13.7 & 31.2 & 13.4 & 35.9 & 14.8 \\
$1680-1779$ & 37.8 & 19.3 & 41.6 & 21.5 & 36.8 & 16.5 & 39.7 & 17.8 \\
$1780-1879$ & 45.0 & 45.0 & 49.6 & 24.2 & 43.7 & 18.7 & 45.6 & 21.0 \\
\hline
\end{tabular}

Source: Thomas H. Hollingsworth, A Demographic Study of the British Ducal Families (1), p. 12.

Table 7. Life expectancy at 20 in families of French and British dukes and peers, and in European ruling families in the 17th-18th centuries

\begin{tabular}{lcccc}
\hline \multirow{2}{*}{ Gender } & $\begin{array}{c}\text { French dukes } \\
\text { and peers }\end{array}$ & $\begin{array}{c}\text { Ruling families } \\
\text { in Europe }\end{array}$ & \multicolumn{2}{c}{ English ducal families } \\
\cline { 2 - 5 } & $1640-1739$ & $1680-1779$ & $1650-1699$ & $1700-1749$ \\
\hline Men & 34.5 & 33.4 & 30.0 & 39.9 \\
Women & 33.6 & 36.2 & 35.4 & 44.2 \\
\hline Total & 34.0 & 34.8 & 32.7 & 42.0 \\
\hline
\end{tabular}

Source: Louis Henry, Claude Levy, "Ducs et pairs,” p. 826.

${ }^{34}$ Bożena Płonka-Syroka, Medycyna w historii i kulturze, Warszawa-Bellerive-sur-Allier: Wydawnictwo DiG, 2016, 246; see also T. Heimarth, "Przełomy w rozwoju nowożytnego położnictwa uwarunkowane nowymi metodami postępowania" in Spoleczno-ideowe aspekty medycyny i nauk przyrodniczych XVIII-XX wieku, ed. Bożena Płonka-Syroka (series: Studia z Dziejów Kultury Medycznej, vol. 5) (Wrocław: Oficyna Wydawnicza Arboretum, 2002): 211+.

${ }^{35}$ Judith Lewis, "'Tis a Misfortune to be a Great Ladie': Maternal Mortality in the British Aristocracy, 1558-1959," Journal of British Studies 37, no. 1 (1998): 31-32.

${ }^{36}$ Lewis, 32. 
The consequences of dying at a relatively young age were varied: social, psychological, economic or demographic, and we have to remember that at the time of the father's death, his offspring were often still underage. In order to determine the consequences of a magnate's death, it is important to consider the average age of marriage and to calculate the average length of marriage - on average, the magnate entered into marriage at the age of 27 or 28 , and on average his marriage lasted about 16 years, which was too short for children, especially sons, to reach adulthood in all magnate relationships. It was very important, especially in the case of sons (a man in a magnate family often decided to marry after his father's death, whether following or disregarding his earlier suggestions as to the choice of wife). The early death of the father often meant that the care of the father's children was left in the hands of his relatives, especially if the widow remarried. In such cases, mothers used to lose custody of their children and thus their emotional bond with them was broken. Hence, one of the consequences of a magnate's death was that his widow had to choose between remarriage and keeping custody of her children and their property. The latter choice meant no further children for many young women. On the other hand, the death of one of the spouses would often result in the remarriage of the other (then, the problem of subsequent marriages and stepfamilies occurred).

It is very important to note that the death of a childless magnate led to the extinction of the family or one of its lines, which resulted in the transference of property to other families or lines. Therefore, the necessity to plan a family policy in such way that the family could be continued was indispensable.

\section{Conclusion}

In conclusion, it should be noted that the life expectancy of magnates in the Grand Duchy of Lithuania and their families was lower than that of the aristocracy in Western European countries.

The average lifespan of magnate females was lower than for European female aristocrats, although the result could have been influenced by high perinatal mortality and may have resulted from a smaller research sample. Magnates had better living conditions (access to high quality food, better housing conditions and higher chances of avoiding contagious diseases), than any other social group, did not usually die in battles (although they were army commanders), but suffered from diseases resulting from prosperity and high social and political position, 
e.g., gout (podagra), obesity ${ }^{37}$ or psychological tensions resulting from their political involvement. Besides, as we have noted above, the standard of medical care was worse than in Western Europe.

\section{Bibliography}

Augustyniak, Urszula. Testamenty ewangelików reformowanych $w$ Wielkim Księstwie Litewskim w XVI-XVIII wieku, Warszawa: Semper, 2014.

Cummins, Neil. "Lifespans of the European Elite, 800-1800." The Journal of Economic History 77, no. 2 (2017): 406-439.

Baten, Joerg, and Tom Keywood. "Elite Violence and Elite Numeracy in Europe from 500 to 1900 CE: A Co-Evolution?" (2019); from C.E.P.R. Discussion Papers, no. 14013.

Baten, Joerg, Mikołaj Szołtysek, and Monica Campestrini. "Girl Power" in Eastern Europe? The Human Capital Development of Central-Eastern Europe in the Seventeenth to Nineteenth Centuries and its Determinants." European Review of Economic History 21, no. 1 (2017): 29-63.

David, Paul, S. Ryan Johansson, and Andrea Pozzi. "The Demography of an Early Mortality Transition: Life Expectancy, Survival and Mortality Rates for Britain's Royals, 1500-1799." University of Oxford Discussion Papers in Economic and Social History 83 (2010): 1-59.

Easterlin, Richard A. "The Worldwide Standard of Living Since 1800.” Journal of Economic Perspectives 14, no. 1 (2000): 7-26.

Feichtinger, Gustav, Maria Winkler-Dworak, Inga Freund, and Alexia Prskawetz. "On the Age Dynamics of Learned Societies-Taking the Example of the Austrian Academy of Sciences." Vienna Yearbook of Population Research, Vienna Institute of Demography, Austrian Academy of Sciences (2007): 107-131.

Furtak, Tadeusz. "Kilka zagadnień z demografii historycznej szlachty polskiej.” Roczniki Dziejów Społecznych i Gospodarczych 4 (1937): 31-58.

Heimrath, Tadeusz. "Przełomy w rozwoju nowożytnego położnictwa uwarunkowane nowymi metodami postępowania." In Społeczno-ideowe aspekty medycyny i nauk przyrodniczych XVIII-XX wieku, edited by Bożena Płonka-Syroka (series: Studia z Dziejów Kultury Medycznej, vol. 5), Wrocław: Oficyna Wydawnicza Arboretum, 2002.

Henry, Louis, and Claude Levy. "Ducs et pairs sous l'Ancien Régime: Caractéristiques démographiques d'une caste." Population 15 (1960): 807-830.

Hollingsworth, Thomas H. "Mortality in the British Peerage Families Since 1600." Population 32, no. 1 (1977): 323-352.

"A Demographic Study of the British Ducal Families." Population Studies 2, no. 1 (1957): 4-26.

${ }^{37}$ Which was then a symbol of the status and virility of a man - see: Jakub Węglorz, Zdrowie, choroba i lecznictwo w społeczeństwie XVI-XVIII wieku (Toruń: Wydawnictwo Adam Marszałek, 2015), 72. 
- "The Demography of the British Peerage." Population Studies vol. 18, no. 2 (1964), (Supplement): 1-108.

Huck, Paul. "Infant Mortality and Living Standards of English Workers During the Industrial Revolution.” Journal of Economic History 55, no. 3 (1995): 528-550.

Kędelski, Mieczysław. "Ewolucja umieralności i trwania życia ludności miasta Poznania w wiekach XIX i XX.” Studia Demograficzne 85 (1986): 3-26.

____. "Piramida wieku i tablice trwania życia ludności Śląska w 1864 roku." Przeszłość Demograficzna Polski 20 (1997): 49-71.

___ “_Umieralność i trwanie życia w Wielkopolsce w latach 1816-1875.” Przeszłość Demograficzna Polski 16 (1985): 109-138.

___ Umieralność i trwanie życia ludności Wielkopolski w XIX wieku. Poznań: Akademia Ekonomiczna w Poznaniu, 1996.

Kuchowicz, Zbigniew. "Jeszcze o zdrowiu i niezdrowiu magnatów XVII-XVIII w." Kwartalnik Historyczny 77, no. 2 (1970): 394-403.

Kuchowicz, Zbigniew. "Społeczne konsekwencje postępującej degeneracji możnowładztwa polskiego w XVII-XVIII w.” Kwartalnik Historyczny 76, no. 1 (1969): 21-43.

Lesiak, Anna. "Kobiety z rodu Radziwiłłów w świetle inwentarzy i testamentów (XVIXVIII w.)." In Administracja i życie codzienne w dobrach Radziwitłów XVI-XVIII wieku ("Fasciculi Historici Novi," vol. IX), edited by Urszula Augustyniak, 119-130. Warszawa: Wydawnictwo DiG, 2009.

Lewis Judith. "'Tis a Misfortune to be a Great Ladie": Maternal Mortality in the British Aristocracy, 1558-1959.” Journal of British Studies 37, no. 1 (1998): 31-32.

Liedke, Marzena. "Demografia rodziny magnackiej w Wielkim Księstwie Litewskim na tle elit zachodnioeuropejskich. Wybrane problemy.” Przeszłość Demograficzna Polski 37, no. 1 (2015): 37-70.

____ " "Rodzina magnacka." In Rodzina i jej gospodarstwo na ziemiach polskich w geografii europejskich struktur rodzinnych do połowy XIX wieku, edited by Piotr Guzowski, and Cezary Kuklo, 173-218. Białystok: Instytut Badań nad Dziedzictwem Kulturowym Europy 2019.

____ "Śmierć magnata, czyli co liczby mogą powiedzieć o konsekwencji śmierci mężczyzny w rodzinie magnackiej w Wielkim Księstwie Litewskim w XVI-XVIII w.” Kwartalnik Historii Kultury Materialnej 60, no. 2 (2012): 271-282.

___ Rodzina magnacka w Wielkim Księstwie Litewskim. Studium demograficznospołeczne. Białystok: Instytut Badań nad Dziedzictwem Kulturowym Europy, 2016.

Lukowski, Jerzy. The European Nobility in the Eighteenth Century. New York: Palgrave Macmillan, 2003.

Peller, Sigismund. "Births and Deaths Among Europe's Ruling Families Since 1500." In Population in History. Essays in Historical Demography, edited by David V. Glass, David E.C. Eversley, 87-101. London: E. Arnold, 1965.

____ . "Mortality, Past and Future." Population Studies 1 (1948), no. 4.

. "Studies on Mortality Since the Renaissance." Bulletin of the History of Medicine part 1: 13 (1943): 422-461, part 2: 16 (1944): 362-281. 
Piasecki, Edmund. Ludność parafii bejskiej (woj. kieleckie) w świetle ksiag metrykalnych z XVIII-XX w. Studium demograficzne. Wrocław-Warszawa: PWN, 1990.

____. "Próba sporządzenia okresowych tablic życia na podstawie ksiąg ruchu naturalnego w XVIII-XX w.” Przeszłość Demograficzna Polski 16 (1985): 139-151.

Płonka-Syroka, Bożena. Medycyna w historii i kulturze, Warszawa-Bellerive-sur-Allier: Wydawnictwo DiG, 2016.

Ragauskienè, Raimonda. Mirties nugalèti nepavyko: Biržu ir Dubingiu Kunigaikšču Radvilu biologine istorija ( $X V$ a. pabaiga-XVII a.), Vilnius: Lietuvos edukologijos universiteto leidykla, 2017.

Rostworowski Emanuel. "Zdrowie i niezdrowie polskich magnatów XVI-XVIII wieku." Kwartalnik Historyczny 76, no. 4 (1969): 865-886.

Steckel, Richard H. "Biological Measures of the Standard of Living." Journal of Economic Perspectives 22, no. 1 (2008): 129-152.

Stojek-Sawicka, Karolina. "Rola i miejsce lekarzy w życiu elit politycznych Rzeczypospolitej w epoce staropolskiej." In Relacje lekarz-pacjent $w$ aspekcie społecznym, historycznym i kulturowym, edited by B. Płonka-Syroka (series: Studia z Dziejów Kultury Medycznej, vol. 10), Warszawa: Wydawnictwo DiG, Oficyna Wydawnicza Arboretum, 2005.

Szołtysek, Mikołaj. Rethinking East-Central Europe: Family Systems and Co-residence in the Polish-Lithuanian Commonwealth. Bern: Peter Lang, 2015.

Szreter, Simon, and Graham Mooney. "Urbanization, Mortality, and the Standard of Living Debate: New Estimates of the Expectation of Life at Birth in Nineteenth-Century British Cities." Economic History Review 51, no. 1 (1998): 84-112.

The Decline of Infant Mortality in Europe 1800-1950. Four National Case Studies, eds. C.A. Corsini. P.P. Viazzo, Florence: Unicef, 1993.

Van Zanden, Jan Luiten, Joerg Baten, Marco Mira d'Ercole, Auke Rijpma, and Marcel P. Timmer. How Was Life? Global Well-being Since 1820, OECD publishing, 2014.

Vielrose, Egon. "Przyczynek do demografii szlachty polskiej." Przegląd Statystyczny 1 (1938): 328-342.

Węglorz, Jakub. Zdrowie, choroba i lecznictwo w społeczeństwie XVI-XVIII wieku, Toruń: Wydawnictwo Adam Marszałek, 2015. 


\section{Death of the Magnate: Life Expectancy of the Magnatery in the Polish-Lithuanian Commonwealth Versus the Demography of the Western European Elites in the Early Modern Age}

\section{Summary}

The main purpose of the text was to obtain the life expectancy of the magnatery in the Polish-Lithuanian Commonwealth and compare it with the results from the Western European elites in the early modern age.

Calculation of the life expectancy parameter values was made on the basis of genealogical facts, such as dates of birth and death of the members of 17 families (365 adult men and 173 adult women). Life tables were constructed starting from the age of 20 . On account of the relatively small number of cases, the entire group was adopted as the cohort constituting the basis for constructing the life tables (extreme dates of birth for the cohort are in the case of men: 1457-1800 and women: 1499-1796). Because there were slight differences that may be observed between the life expectancy of magnates from the Kingdom of Poland and the Grand Duchy of Lithuania, I decided to calculate 95\% confidence intervals from my sample. Then I compared the value for the life expectancy of the magnatery with the value for the same parameter of the Western European aristocracy.

My database was constructed using information from genealogical works and published materials referring to particular families, biographies of particular people, and also biographies published in the Polish Biographic Dictionary, monographs of families, articles dedicated to these families and their members, as well as already-published sources, such as wills, letters and memoirs. Certain data were complemented or corrected as a result of an archive query.

Male life expectancy at the age of 20 was almost 33 years, and at the age of 50 , 14.5. On average, women who reached their 20 s carried on living for a further 31 years, and after reaching the age of 50, still had 19 years to go. Differences in life expectancy between men and women from different parts of the country turned out to be minor. The results of calculating 95\% confidence intervals from my sample are not statistically significant. However, we can see bigger differences in relation to the Western European elites. Studies conducted by Western European historical demographers showed longer life expectancy for both men and women, especially in the second half of the 17 th and 18 th centuries.

The lower life expectancy of the magnatery might have been the result of worse medical care, and in a broader sense, a lower level of human capital.

The very small differences in life expectancy between men and women from different parts of the Polish-Lithuanian Commonwealth may have resulted from the small amount of data collected and, on the other hand, of a similar lifestyle and economic and environmental conditions. In addition, they were related to each other as a result of marriage between representatives of both states of the Polish-Lithuanian Commonwealth. 


\section{Śmierć magnata. Oczekiwana długość życia magnaterii w Rzeczypospolitej Obojga Narodów a demografia elit zachodnioeuropejskich w epoce wczesnonowożytnej}

\section{Streszczenie}

Celem artykułu było ustalenie oczekiwanej długości życia magnaterii w Rzeczypospolitej Obojga Narodów i porównanie uzyskanych wyników z tymi, które obliczono dla elit zachodnioeuropejskich w okresie wczesnonowożytnym.

Obliczenia wartości tego parametru dokonano na podstawie faktów genealogicznych, takich jak daty urodzin i śmierci przedstawicieli 17 rodów (356 dorosłych mężczyzn i 173 dorosłe kobiety). Tablice wymieralności skonstruowano, zaczynając od wieku 20 lat. Ze względu na relatywnie niedużą liczbę przypadków, podstawą konstrukcji tablic była cała analizowana grupa mężczyzn i kobiet (skrajne daty urodzin dla kohorty w przypadku mężczyzn to 1457-1800, a w odniesieniu do kobiet - 1499-1796). Ponieważ zaobserwowano niewielkie różnice w oczekiwanej długości życia magnatów z Korony i Wielkiego Księstwa Litewskiego, zdecydowano obliczyć 95\% przedziały ufności. Następnie dokonano porównania oczekiwanej długości życia magnaterii z wartością tego parametru obliczonego dla elit zachodnioeuropejskich.

Baza danych została skonstruowana na podstawie informacji zaczerpniętych z prac genealogicznych i opublikowanych materiałów odnoszących się do poszczególnych rodzin, z biografii poszczególnych osób i monografii rodzin, biogramów z Polskiego Słownika Biograficznego, artykułów poświęconych danym rodzinom i ich przedstawicielom oraz źródeł historycznych już opublikowanych, takich jak testamenty, listy i pamiętniki. Dane te uzupełniono lub skorygowano w wyniku kwerendy archiwalnej.

Oczekiwana długość życia magnatów w wieku 20 lat wyniosła prawie 33 lata, a w wieku 50 lat - 14,5 lat. Dwudziestoletnie kobiety zaś żyły jeszcze średnio 31 lat, a te w wieku 50 lat - jeszcze 19 lat. Różnice między oczekiwaną długością życia przedstawicieli obu części Rzeczypospolitej Obojga Narodów były niewielkie. Jednak możemy obserwować większe różnice w oczekiwanej długości życia miedzy magnaterią a elitami zachodnioeuropejskimi, na korzyść tych ostatnich, szczególnie w drugiej połowie XVII i w XVIII wieku.

Niższa oczekiwana długość życia magnaterii mogła wynikać z gorszej niż na Zachodzie opieki medycznej, a w szerszym ujęciu - z niższego poziomu kapitału ludzkiego. Niewielkie różnice w wartości tego parametru między przedstawicielami Korony i Wielkiego Księstwa Litewskiego mogły wynikać z jednej strony z niewielkiej próby badawczej, a z drugiej - z podobnego stylu życia oraz warunków ekonomicznych i środowiskowych. Ponadto przedstawiciele obu głównych części Rzeczypospolitej byli ze sobą spokrewnieni na skutek koligacji małżeńskich. 